\title{
Load Factor Assessment and Evaluation on the performance MIMO WCDMA Rayleigh Interference Channel
}

\author{
Affum Emmanuel A.K \\ Department of Electrical Engineering. \\ Kwame Nkrumah University of Science \& \\ Technology \\ Kumasi, Ghana
}

\author{
Danso Ansong, Ed \\ Department of Computer Science \& I. T \\ Valley View University \\ Oyibi - Accra, \\ Ghana
}

\begin{abstract}
Multiple Access Interference (MAI) is interference caused by the presence of other users in the cell. Conventional signal detectors detect only single user's signal. When there are multiple users in the same environment, the conventional detectors treat other users' signals as noise or interference. MAI affects system capacity and system performance. When there are more users, the MAI is high besides the performance is also affected by the near-far problem. This paper seeks to analyse the Capacity of MIMO WCDMA under various Cell Load factors. With cell loading factor of $6 \%$ and signal to noise ratio of $10 \mathrm{~dB}$ the $4 * 4$ MIMO systems performed better. Besides for a cell load of $50 \%$ and a cell range of $1.02 \mathrm{~km}$ the allowable path loss without MUD was around $98.45 \mathrm{~dB}$
\end{abstract}

\section{General Terms}

WCDMA; Universal mobile Telecommunications System; UMTS

\section{Keywords}

Load Factor, Multi Input Multi Output ; MIMO; Detection, Wideband Code Division Multi Access

\section{INTRODUCTION}

The Code Division Multiple Access (CDMA) system is an interference limited system in which link performance depends on the ability of the receiver to detect a signal in the presence of interference. Therefore, the key issue in a CDMA network design is to minimize multiple access interference that can be achieved by critical power control. Interference on the voice channels causes cross talk where the subscriber hears interference in the background due to an undesired transmission, on control channels, interference leads to missed and blocked calls due to errors in the digital signaling. Interference is more severe in the urban areas, due to the greater RF noise floor and the large number of base stations and mobiles [1] and therefore, has been recognized as a major bottleneck in increasing capacity responsible for dropped calls [2]. Sources of interference include another mobile in the same cell, a call in progress in a neighboring cell, other base stations operating in the same frequency band, or any cellular system which inadvertently leaks energy into the cellular frequency band. In [3], Heiska analyzed capacity reduction of WCDMA downlink in the presence of interference from adjacent narrow-band system by taking into account different downlink interference mechanisms such as wide-band noise from the transmitter as well as adjacent channel interference, intermodulation, and cross-modulation originating in the mobile receiver, and concluded that capacity per cell is sensitive to the cell size, and therefore, very careful network planning is needed in order to operate the WCDMA networks efficiently. The Interference Performances, when WCDMA and HSDPA coexist as analyzed by Pei Li in [4], provided simulation results indicating that the system performance in the hybrid cells is better than the pure macro cell for WCDMA and HSDPA. After investigation of WCDMA interoperator adjacent channel interference, Joyce et al [5], proposed a number of measures which both operators and vendors should take to avoid deadzones in an operational WCDMA network.

Extensive studies have been done by Gao Peng and the group [6] where they analyzed the interference between WCDMA and WIMAX systems to evaluate the impact of inter-system interference produced by coexistence of systems in the same geographical area in adjacent frequency and concluded that WCDMA and WiMax systems could coexist and gave the proposals of interference mitigation method in the case of coexistence of two systems. Results on the other-cell to owncell interference values and traffic capacity for dedicated indoor WCDMA systems were presented in [7]. Also, Kiiskila and the group in [8] discussed receiver complexity and presented optimal and suboptimal spatial maximum a posteriori receivers in a concatenation of Linear Minimum Mean Square Error (LMMSE) equalizer structure for Multiple-Input-Multiple-Output (MIMO) Wideband Code Division Multiple Access (WCDMA) systems, and further, proposed that in frequency selective fading channels where LMMSE part mitigates the Multiple Access Interference (MAI) and Inter-Antenna Interference (IAI) is achieve by the spatial MAP or its approximation. Potential GPRS 900/180$\mathrm{MHz}$ and WCDMA $1900-\mathrm{MHz}$ Interference to Medical Devices were also investigated by Iskra et al and compared the potential for interference to medical devices from Radio Frequency (RF) fields radiated by GSM 900/1800-MHz, General Packet Radio Service (GPRS) 900/1800-MHz, and Wideband Code Division Multiple Access (WCDMA) 1900$\mathrm{MHz}$ handsets. Performance analysis of MQAM for MIMO WCDMA systems in fading channels has extensively studied [10] with authors developing an analytical framework that could handle an arbitrary number of transmit and receive antennas in both open-loop and closed-loop systems with numerical results showing that the system could achieve significant performance improvement by using the combined transmit and receive antenna diversity.

The above analyses and importance notwithstanding, WCDMA needs further investigation especially in the context of its performance in coexistence with other systems. In this context the uplink WCDMA was analyzed in Raleigh fading channel. Specifically we present an accurate BER analysis of a MIMO uplink WCDMA system in multiple interference 
employing Maximum Mobile Transmitted Power and Equivalent Isotropic radiated power (EIRP) of $21 \mathrm{dBm}$ and $18 \mathrm{dBm}$ respectively with emphasis on the interference from adjacent cells. The paper further presents simulation results to support the theoretical analysis on reverse link capacity analysis in terms of cell loading factor.

Organization: In section II, the uplink and downlink load factors and efficiency of multiuser receiver of CDMA system are analyzed. Section III focuses on the MIMO system. Intercell interference and the reverse link capacity in singlecell and multi systems are analyzed in section IV. In section $\mathrm{V}$, numerical and simulation results are shown and discussed, while section VI concludes this paper.

\section{CDMA UPLINK AND DOWNLINK LOAD FACTORS}

\subsection{Uplink Load Factor}

The $\left(E_{b} / I_{t}\right)$ for the $i t h$ user is expressed as

$$
\left(\frac{E_{b}}{I_{t}}\right)_{i}=\frac{\beta_{c}}{\beta_{i} \vartheta_{i}} \cdot \frac{R_{i}}{\left(I_{\text {total }}-R_{i}\right)}
$$

Where $\beta_{c}$ is the chip rate, $R_{i}$ is the received signal power from ith user, the channel activity factor of ith user is represented by $v_{i} . \beta_{i}$ is the bit rate of the $i t h$ and $I_{\text {total }}$ as total received power including thermal noise power at the base station. Let $R_{i}=\varphi_{i}$. I total where $\varphi_{i}$ is the load factor of the ith connection [1], then

$$
\varphi_{i}=\frac{1}{1+\frac{\beta_{c}}{\left(\frac{E_{b}}{I_{t}}\right)_{i} \cdot \beta_{i} \vartheta_{i}}}
$$

The total received interference [1], without the thermal noise $N_{T}$, can be expressed as the sum of the total received powers from all $M$ user in the same cell $I_{\text {total }}-N_{T}=$ $\sum_{i=1}^{M} R_{i}=\sum_{i=1}^{M} \varphi_{i} . I_{\text {total }}$. If noise rise of the entire system is expressed as noise rise $=I_{\text {total }} / N_{T}=1 /\left(1-\sum_{i=1}^{M} \varphi_{i}\right)$ $=1 /\left(1-\delta_{u l}\right)$, where $\delta_{u l}$ is the uplink load factor and is expressed as $\delta_{u l}=\sum_{i=1}^{M} \varphi_{1}$. When $\delta_{u l}$ approaches 1 , the corresponding noise-rise approaches infinity and the system reaches its pole capacity. If $\gamma$ is the interference factor due to other cells, in terms of interferences from other cell, the interference factor can be expressed as

$$
\gamma=\frac{\text { other cell interference }}{\text { own cell interference }}
$$

Then uplink load factor can then be written as

$$
\begin{gathered}
\delta_{u l}=(1+\gamma) \cdot \sum_{i=1}^{M} \varphi_{i} \\
=(1+\gamma) \cdot \sum_{i=1}^{M} \frac{1}{1+\frac{\beta_{C}}{\left(E_{b} / I_{t}\right)_{i} \cdot \beta_{i} \cdot \vartheta_{i}}}
\end{gathered}
$$

The load equation at (4) predicts the amount of noise-rise over thermal-noise due to interference, and also could be used to make semi analytical predictions of the average capacity of CDMA cell and finally could also be employed in predicting cell capacity and planning noise-rise for dimension purposes [1]. The noise-rise is equal to $-10 \log \left(1-\delta_{u l}\right)$. The interference margin in the link budget must be equal to the maximum planned noise-rise

\subsection{Multiuser Receiver Efficiency}

The interference caused by the presence of other users in the cell is called Multiple Access Interference [MAI]. Conventional signal detectors detect only single user's signal. When there are multiple users in the same environment, the conventional detectors treat other users' signals as noise or interference. MAI affects system capacity and system performance. When there are more users, the MAI is high [1]. The system performance is also affected by the near-far problem. The use of multiuser detection techniques has also been suggested in the WCDMA UMTS system. Multiuser detection (MUD) and interference cancellation (IC) technique improve the system performance by canceling the intercell interference. MUD also known as co channel interference suppression or multiuser demodulation exploits the considerable structure of the multiuser interference in order to increase the efficiency with which channel resources are employed [11].

Since MUD efficiency varies in different radio environment, the capacity improvement attainable by MUD is not fixed. The impact of MUD on coverage introduces a new variable to the network planning process, since MUD efficiency need to be taken into account in the coverage design. The efficiency of MUD is estimated from the load that can be supported with a specified $E_{b} / I_{t}$ value with a multiuser received. In the analysis, the number of users with a RAKE receiver is represented by $M_{R A K E}$ and those with a MUD receiver by $M_{M U D}$. The efficiency of MUD receiver also denoted by $\eta$ at a give $E_{b /} I_{t}$ is [12] $M_{R A K E}=(1-$

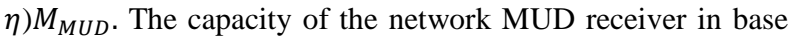
transceiver station (BTS) in terms received signal power $R_{s p}$, power control efficiency $\gamma_{c}$ is expressed as

$$
\frac{E_{b}}{I_{t}}=\frac{R_{s p} \cdot P_{a} \cdot \gamma_{c}}{(1-\eta) \psi_{\text {intra }}+\psi_{\text {inter }}+N_{o}}
$$

Where $N_{o}$ is thermal noise, $\psi_{\text {intra }}$ is the intracell interference from own cell mobiles, $\psi_{\text {inter }}$ is the interference from the mobiles not connected to this particular base station, and $P_{a}$ is the processing again. But $\mu=\psi_{\text {intra }} / \psi_{\text {intra }}+\psi_{\text {inter }}$ and hence $\left.\psi_{\text {inter }}=((1-\mu) / \mu)\right)$. $\psi_{\text {intra }}$. Therefore, substituting it into Equation (5) and neglecting the effect of thermal noise. Equation (5) becomes

$$
\frac{E_{b}}{I_{t}}=\frac{R_{s p} \cdot P_{a} \cdot \gamma_{c}}{(1-\eta)(M-1) R_{s p}+\left(\frac{1-\mu}{\mu}\right) M \cdot R_{s p}}
$$

Where $\mathrm{M}$ is the number of users associated with the BTS. Further solving equation (6) for M, will result in

$$
M=\frac{\mu\left[\psi_{c} \cdot P_{a} \cdot\left(E_{b} / I_{t}\right)^{-1}+(1-\mu)\right]}{1-\mu \eta}
$$

In an unloaded network, the uplink limits the achievable range and coverage, as the maximum transmission power of the mobile station is lower compared with the maximum transmission power of the base station in the downlink. In a loaded network, the downlink may limit the range if there is more load and thus more interference in the downlink than the uplink. The received signal-to-interference ratio at the base station

is given

as 


$$
\frac{E_{b}}{I_{t}}=\frac{E_{b, \text { loaded }}}{\varphi_{\text {intra }}+\varphi_{\text {inter }}+N_{o}}
$$

Where $E_{b}$ is the received energy per bit, $\psi_{\text {intra }}$ is the intracell interference from own cell mobiles, $\psi_{\text {inter }}$ is the interference from the mobiles not connected to this particular base station, and $N_{o}$ is the thermal noise. In case of an unloaded network $\psi_{\text {intra }}=0, \psi_{\text {inter }}=0$, and the required $E_{b} / N_{o}$ for range calculations is equal to $E_{b} / I_{t}$. In the loaded network, the fraction of own-cell interference from total interference is defined as

$$
\varpi=\frac{\psi_{\text {intra }}+S}{\psi_{\text {intra }}+\psi_{\text {inter }}+S}
$$

Where $S=E_{b} / P_{a}$ the received signal is power from one user and $P_{a}$ is the processing gain. $\varpi$ depends upon propagation environment. The higher the path-loss attenuation factor, the higher the $\varpi . \psi_{\text {inter }}$ can be expressed in term of $\psi_{\text {intra }}$ as

$$
\psi_{\text {inter }}=\psi_{\text {intra }}\left(\frac{1}{\varpi}-1\right)+\frac{E_{b, \text { loaded }}}{P_{a}}\left(\frac{1}{\varpi}-1\right)
$$

but

$$
\psi_{\text {intra }}=(M-1) \frac{E_{b, \text { loaded }}}{P_{a}}
$$

therefore,

$$
\begin{aligned}
\left(\psi_{\text {inter }}+\psi_{\text {intra }}\right)=\left(\frac{M}{\varpi}-1\right) \frac{E_{b, \text { loaded }}}{P_{a}} & \begin{aligned}
\frac{E_{b, \text { loaded }}}{I_{t}} & =\frac{E_{b, \text { loaded }}}{\left(\frac{M}{\varpi}-1\right) \frac{E_{b, \text { loaded }}}{P_{a}}+N_{o}} \\
& =\left(\frac{E_{b}}{N_{o}}\right)_{\text {unloaded }}
\end{aligned}
\end{aligned}
$$

Solving the required $E_{b} / N_{o}$ in the loaded case gives

$$
\left(\frac{E_{b}}{N_{o}}\right)_{\text {loaded }}=\frac{1}{\left(\frac{E_{b}}{N_{o}}\right)_{\text {unloaded }}^{-1}-\left(\frac{M}{\varpi}-1\right) \frac{1}{P a}}
$$

The effect of the MUD receiver can be taken into account by using the efficiency of the MUD $\eta$ as a measure of performance of the MUD receiver. With MUD receiver, the intracell interference $\psi_{\text {intra,MUD }}$ can be written as

$$
\begin{gathered}
\psi_{\text {intra }, M U D}=(1-\eta) \psi_{\text {intra }} \\
=(1-\eta)(M-1) \frac{E_{b}}{P_{a}}
\end{gathered}
$$

and

$$
\begin{gathered}
\psi_{\text {inter }}= \\
\left(\frac{1}{\varpi}-1\right)(1-\eta)(M-1) \frac{E_{b}}{P_{a}}+\frac{E_{b}}{P_{a}}\left(\frac{1}{\varpi}-1\right)
\end{gathered}
$$

the total interference will be

$\psi_{\text {inter }}+\psi_{\text {intra,MUD }}$

$$
=\frac{E_{b, \text { loaded }}}{P_{a}}\left[\frac{M(1-\eta)+\eta}{\varpi}-1\right]
$$

The required $E_{b} / I_{t}$ in the loaded network with MUD receiver becomes

$$
=\frac{\left(\frac{E_{b}}{N_{o}}\right)_{\text {loaded,MUD }}}{\left(\frac{E_{b}}{N_{o}}\right)_{\text {unloaded }}^{-1}-\left[\frac{M(1-\eta)+\eta}{\varpi}-1\right] \frac{1}{P_{a}}}
$$

(9) The transmitted power from a mobile is given as

$$
S_{T X, M S}=\frac{E_{b}}{N_{o}}+R_{b}+N_{f}+k T-G_{H O}-G_{M S}-G_{B S}
$$

In Equation (19) above all the terms are the same except for $E_{b} / N_{o}$, regardless of the base station receiver algorithm. $S_{T X, M S}$ is determined only from the $E_{b} / N_{o}$ requirement. The decrease in the required transmission power with MUD receiver is thus given as

$$
\begin{gathered}
\frac{S_{T X, M S}}{S_{T X, M S, M U D}}=\frac{\left(\frac{E_{b}}{N_{o}}\right)_{\text {loaded }}}{\left(\frac{E_{b}}{N_{o}}\right)_{\text {unloaded }, M U D}} \\
=\frac{\left(\frac{E_{b}}{N_{O}}\right)_{\text {loaded }}-\left[\frac{M(1-\eta)+\eta}{\varpi}-1\right] \frac{1}{P_{a}}}{\left(\frac{E_{b}}{N_{o}}\right)^{-1} \text { unloaded }-\left[\frac{M}{\varpi}-1\right] \frac{1}{P a}}
\end{gathered}
$$

\section{FREQUENCY SELECTIVE MIMO \\ CHANNEL}

The general expression of frequency-selective MIMO channel indicates $N_{I}$ signals $x_{\mu}[k], 1 \leq \mu \leq N_{I}$ from the input of the system at each time instant $k$ and we obtain $N_{O}$ output. Therefore, the $v^{\text {th }}$ output at time instant $k$ can be expressed as [13]

$$
y_{v}[k]=\sum_{\mu=0}^{N_{I}} \sum_{\kappa=0}^{L_{t}-1} h_{v, \mu}[K, \kappa] \cdot x_{\mu}[K-\kappa]+n[K]
$$

where $L_{t}$ denotes the largest number of taps among all the contributing channels. The channel matrix has the form [14].

$$
H[K, \kappa]=\left[\begin{array}{ccc}
h_{1,1}[K, \kappa] & \cdots & h_{1, N_{I}}[K, \kappa] \\
\vdots & \ddots & \vdots \\
h_{N_{O}, 1}[K, \kappa] & \ldots & h_{N_{O}, N_{I}}[K, \kappa]
\end{array}\right]
$$

\subsection{Receiver Processing}

If coherent single-user matched filter is used where the receiver is assumed to know the fading coefficients of the user of interest and the transmitted signal from each antenna $K=1$

15], then an antenna will receive

$$
\begin{gathered}
y_{1}=A_{11} b s_{11}(t)+\sigma n_{1}(t) \\
\vdots \\
y_{D}=A_{D 1} b s_{D 1}(t)+\sigma n_{D}(t)
\end{gathered}
$$

Optimum decision rule selects $b \in\{-1,1\}$ that minimizes

$$
\int_{0}^{T} \sum_{d=1}^{D}\left|y_{d}(t)-A_{d 1} b s(t)_{d 1}\right|^{2} d t
$$


According to the optimum decision rule [10] the inner product of the $y(t)$ and $s(t)$ is the sufficient statistic [14]. This means that the optimum rule decision for a single -user case is expressed as

$$
\hat{b}=\operatorname{sgn}\left(\mathfrak{R}\left\{A \sum_{d=1}^{D} A_{d 1} y_{d 1}^{*}\right\}\right)
$$

(25)

Therefore, the probability of error of a MIMO system could be expressed as [14].

$$
P_{k}^{D c}(\sigma)=E\left[Q\left(\frac{\sum_{d=1}^{D}\left|A_{d k}\right|^{2}}{\sum_{d=1}^{D}\left|A_{d k}\right|^{2}\left(\sigma^{2}+\sigma_{I_{U W B}^{(k)}}^{2}\right)}\right)\right]
$$

\section{INTERCELL INTERFERENCE}

\subsection{Link Capacity}

Considering an omnidirection cell site serving a given set of mobiles, if mobiles are divided into two groups which are mobiles that are powered up and mobiles that are not powered up, the mobiles that are powered up are further, divided into four subgroups: Active and transmitting mobiles, Active but not transmitting mobiles (mobiles in non conversational mode), Idle and transmitting (mobiles in access mode) and Idle and not transmitting (mobiles in non access mode) [1].

Assume there are $\mathrm{M}$ mobiles transmitting at a given time in a cell. In a CDMA environment for each mobile, there are $(\mathrm{M}-$ 1) interferers. At the cell site, the average signal power received from the ith mobile is $S_{r i}$. This signal power provides bit energy equal $E_{b}=S_{r i} / R$ where, $\mathrm{R}$ is the mobile transmission rate in bps. The thermal noise power is $N_{0} B_{w}$, where $N_{0}$ is the thermal noise power spectral density $(p s d)$, and $B_{w}$ is the spreading bandwidth. The average interference (psd) at the base station is expressed as

$$
I_{0}=\frac{1}{B_{w}} \sum_{i=1}^{M-1} V_{f} . S_{r i}
$$

Where, $V_{f}=$ channel activity factor.

In equation (28), assuming a perfect control in the reverse link and that the signals transmitted from all the mobiles arrived at the base station with the same received power. i.e. $S_{r i}=S$ for all values of $i$ (i.e. $(1 \leq i \leq M-1)$. The total interference and thermal noise ( $p s d)$ will be

$$
I_{t}=I_{0}+N_{0}=\frac{1}{B_{w}} \sum_{i=1}^{M-1} V_{f} \cdot S_{r i}+N_{0} .
$$

Recognizing that $S_{r i}=S, I_{t}$ then becomes

$$
I_{t}=\frac{(M-1) \cdot V_{f} \cdot S}{B_{w}}+N_{0}
$$

The $E_{b} / I_{t}$ will be given as,

$$
\begin{aligned}
\frac{E_{b}}{I_{t}} & =\left(\frac{B_{w}}{r}\right) \cdot \frac{s}{\left[N_{0} B_{w}+(M-1) \cdot V_{f} \cdot S\right]} \\
& =G_{p} \cdot \frac{s}{\left[N_{0} B_{w}+(M-1) \cdot V_{f} \cdot S\right]}
\end{aligned}
$$

Where $G_{p}=$ processing gain $=B_{w} / r$. The signal strength, $\mathrm{S}$ in $d B$ as,

$$
\begin{gathered}
S=P_{m}+G_{m}+G_{b}+G_{d v}+G_{\text {sho }}+L_{p}+M_{\text {fade }} \\
+L_{\text {body }}+L_{\text {pent }}+L_{\text {cable }}
\end{gathered}
$$

Where

$G_{m}=$ transmit antenna gain of the mobile $(d B)$

$G_{b}=$ receive antenna gain of base station $(d B)$

$G_{d v}=$ base station antenna diversity $(d B)$

From (30)

$$
M=1+G_{p} \cdot\left[\frac{1}{\left(E_{b} / I_{t}\right) \cdot v_{f}}\right]-\frac{N_{0} \cdot B_{w}}{S \cdot v_{f}}
$$

also,

$$
S=\frac{\left(E_{b} / I_{t}\right) \cdot N_{0}}{\frac{1}{R}-\frac{(M-1) v_{f}\left(E_{b} / I_{t}\right)}{B_{w}}}
$$

Let $\beth$ represent the interference factor from other cells (31) can be expressed as

$$
G_{p} \cdot \frac{S}{\left[N_{0} B_{w}+(M-1) \cdot V_{f} \cdot S(1+ב)\right]}
$$

Include an imperfect power factor, $\varphi$ and rewrite equation (33) as,

$$
G_{p} \cdot \frac{S}{B_{w} \cdot N_{0}+(M-1) \cdot V_{f} \cdot\left(\frac{S}{\varphi}\right) \cdot(1+ב)}
$$

Solving equation (35) for $M$, we get,

$$
M=1+G_{p} \cdot\left[\frac{\varphi}{\left(\frac{E_{b}}{I_{t}}\right) \cdot V_{f} \cdot(1+\beth)}\right]-\frac{N_{0 .} B_{w} \cdot \varphi}{S \cdot V_{f} \cdot(1+\beth)}
$$

Solving equation (35) for $S$, we get

$$
S=\frac{\frac{E_{b}}{I_{t}} \cdot N_{0 .}}{\frac{1}{R}-\frac{(M-1) \cdot V_{f} \cdot(1+\beth) \frac{E_{b}}{I_{t}}}{B_{w} \cdot \varphi}}
$$

From equation (36), the maximum value of $M$ is,

$$
M_{\max }=1+G_{p} \cdot\left[\frac{\varphi}{\left(\frac{E_{b}}{I_{t}}\right) \cdot V_{f} \cdot(1+\beth)}\right]
$$

$\max$ is called the pole point or asymptotic cell capacity that $\ldots$ achieved as $S \rightarrow \infty$. For simplification, neglecting 1 and rewriting equation (38) gives,

$$
M_{\max } \approx G_{p} \cdot\left[\frac{\varphi}{\left(\frac{E_{b}}{I_{t}}\right) \cdot V_{f} \cdot(1+\beth)}\right]
$$


equation (33) can further be expressed as,

$$
\frac{S / \varphi}{N_{0} B_{w}}=\frac{1}{M_{\max } \cdot V_{f} \cdot(1+\beth) \cdot(1-\rho)}
$$

where, $\quad \rho=\frac{M}{M_{\max }}=$ cell loading factor.

\section{RESULT \& DISCUSSIONS}

In this study, a maximum cell loading factor of $18 \%$ and signal to noise ratio of $30 \mathrm{~dB}$ were used for the capacity analyses. With $M$ mobiles of 20 and received antenna gain of the base station of $9 d B$ and with maximum mobile transmitted power and equivalent isotropic radiated power of $18 \mathrm{dBm}$ and $21 \mathrm{dBm}$ respectively, the bits per second performance for the $4 * 4$ MIMO System shown in figure 1 for $30 \mathrm{~dB}$ is around $35 \mathrm{bps}$ that overwhelmed the other systems.

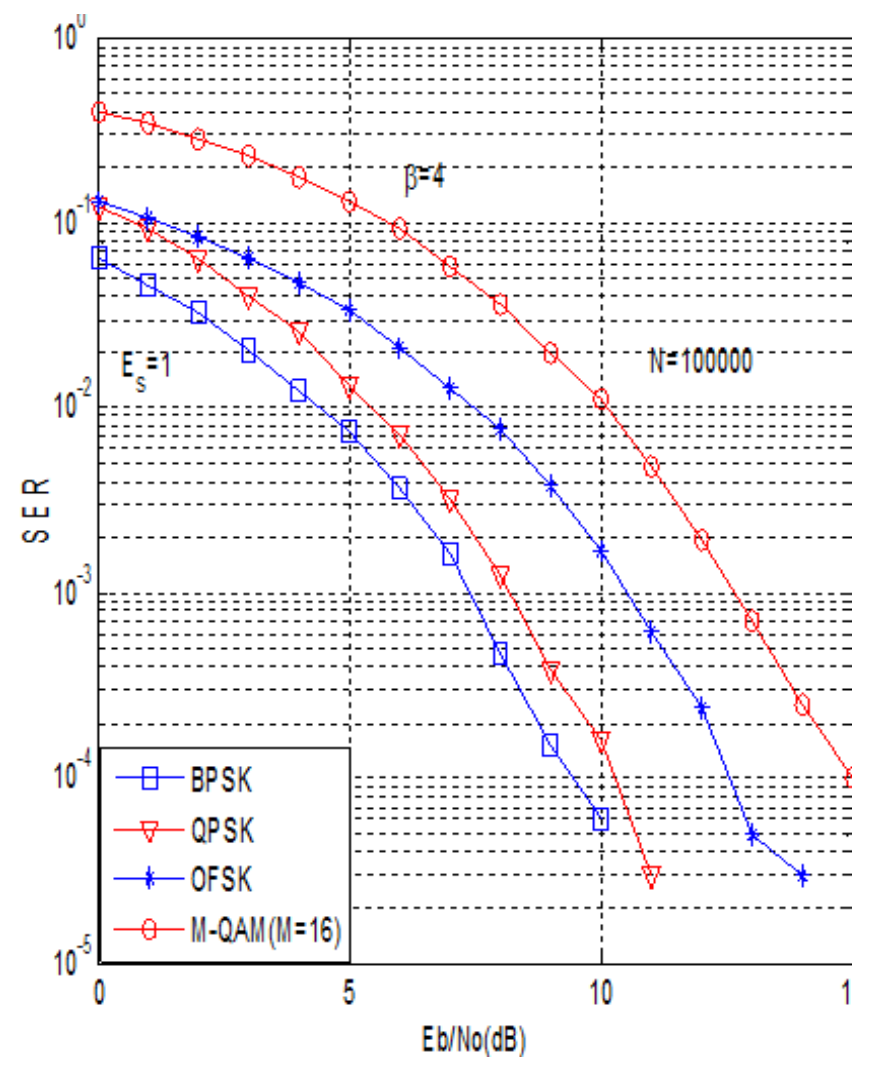

Figure 1 Performances of modulation schemes in WCDMA with $18 \%$ Cell Load factor with $\beta=4$

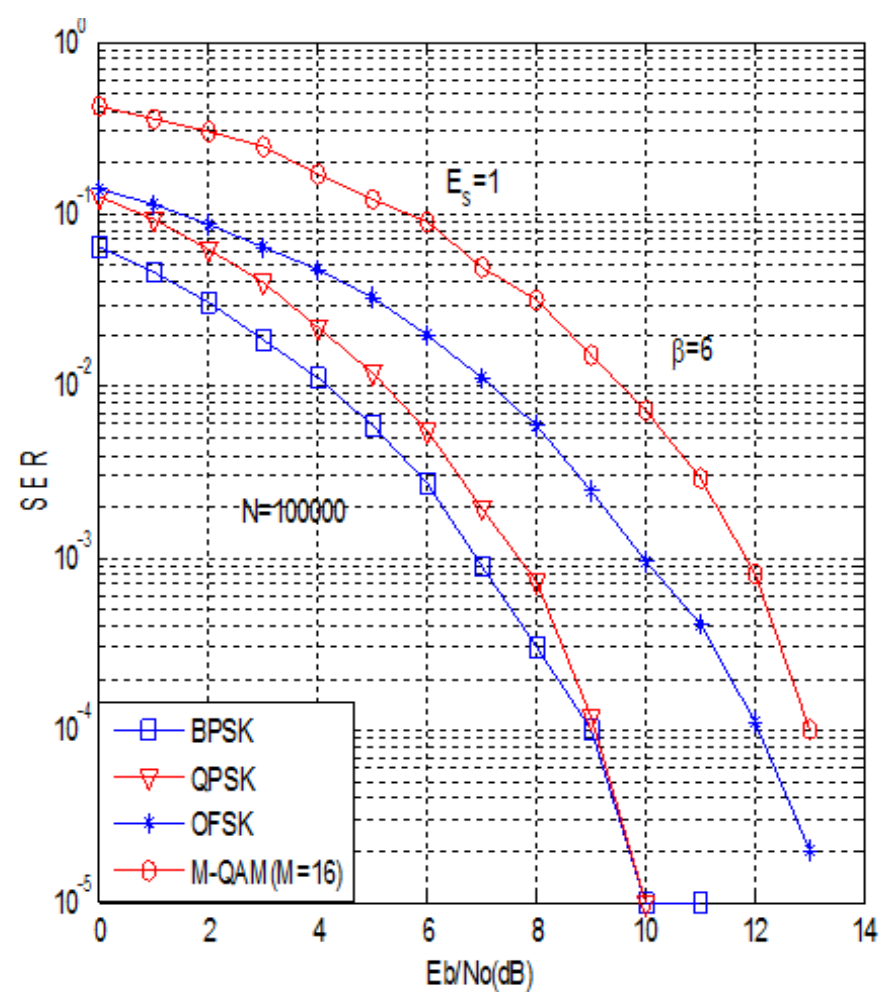

Figure 2 Performances of modulation schemes in WCDMA with $18 \%$ Cell Load factor with $\beta=6$

Figure 2 also provides the BER analysis of the various systems in multiple interferers. It was also realized that as the cell range and allowable path loss in $d B$ decreases the cell load increases dramatically. Interestingly, similar results were obtained in [1] and further observed that base station multiuser detection (MUD) receiver can provide good coverage even with high system load after initial deployment and, finally, concluded that the effect of MUD on cell range depends on propagation environment.

\section{CONCLUSION \& FUTURE WORK}

Analyses of coverage of a loaded and unloaded WCDMA network conducted in this paper revealed that the propagation environment affects the cell range with a given cell loading. Furthermore, for efficient CDMA operation the spectrum must be cleared in a sufficient guard band and guar zone. With cell loading factor of $6 \%$ and signal to noise ratio of $10 \mathrm{~dB}$ the $4 * 4$ MIMO systems performed better. Besides for a cell load of $50 \%$ and a cell range of $1.02 \mathrm{~km}$ the allowable path loss without MUD was around $98.45 \mathrm{~dB}$ Also, spectrum monitoring is highly recommended as early as possible in the CDMA system since it is tedious to identify the source of external interference. Intermodulation interference, adjacent and co-channel interference could be considerd in further studies. With path loss with MUD at a cell range of $1.357 \mathrm{~km}$ was around $110.67 \mathrm{~dB}$. 


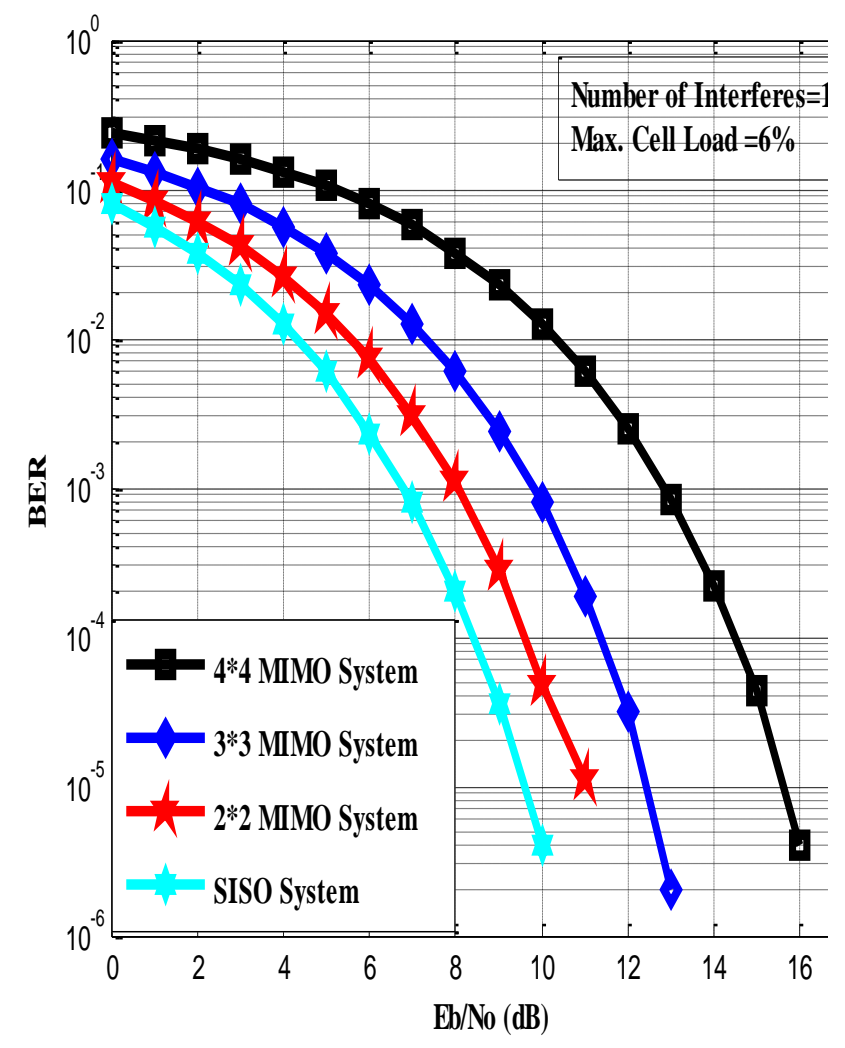

Figure 3 BER performance of WCDMA in Multiple

\section{REFERENCES}

[1] Vijay Garg, K., 2002 “Wireless Networks Evolution $2 G \& 3 G$ ” Prentice Hall PTR, New Jersey

[2] Theodore Rappaport, S., 2002 "Wireless Education, Communications" Second Edition, Pearson Inc, New Jersey

[3] Heiska, K., Posti, H., Muszynski, P., Aikio, P., Numminen, J., Hamalainen, M. 2002 "Capacity Reduction Of WCDMA Downlink in the Presence of Interference from Adjacent Narrow-Band System" Vehicular Technology, IEEE Transactions, vol. 51 , pp. 37

[4] Pei Li, 2006 "The Interference Performances when WCDMA and HSDPA Coexist", Information and Communication Technologies, IEEE, vol. 2, pp. 2450, Damascus.

[5] Joyce, R.M., Graves, B.D., Osborne, I.J., Griparis, T., Conroy, G.R. 2003 "An investigation of WCDMA inter-operator adjacent channel interference", 3G Mobile Communication Technologies, 4th International Conference, IEEE, pp. 149, 25-27

[6] Gao Peng, Tu Guofang, Fang Yuan,Liang Shuangchun 2009. "The analysis of the interference between WCDMA and WIMAX systems", Communications Technology and Applications, ICCTA '09. IEEE International Conference, pp. 180, Beijing,

[7] Schuh, R.E., Andersson, R., Stranne, A., Sommer, M., Karlsson, P. 2003 "Interference analysis for dedicated indoor WCDMA systems", Vehicular Technology Conference, IEEE, vol.2, pp. 992, 690

[8] Kiiskila, K., Hooli, K., Ylioinas, J., Juntti, M., 2005 "Interference resistant receivers for WCDMA MIMO downlink", Vehicular Technology Conference, VTC 2005-spring, IEEE, vol. 2, pp. 836

[9] Iskra, S., Thomas, B.W., McKenzie, R., Rowley, J. 2007 "Potential GPRS 900/180-MHz and WCDMA 1900-MHz Interference to Medical Devices", Biomedical Engineering, IEEE Transactions, vol. 54, issues 10, pp. 1858

[10]Fuyong $\mathrm{Xu}$, Guangqiu Li, 2005 "Performance analysis of MQAM for MIMO WCDMA systems in fading channels", Communications, Circuits and Systems International Conference IEEE, vol. 1, pp. 207

[11]Sergio, V. "Multiuser Detection" The Press Syndicate of the University of Cambridge, Pit Building Trumpington Street, Cambridge, CB2, 1RP, United Kingdom.

[12] Ojanpera, T., and Prasad, R., 1998. "Widband CDMA for Third Generation Mobile Communication" Artech House, Boston,

[13]Foerster J. "Channel modeling sub-committee report final", IEEE 802.15-02/490 (see http://ieee802.org/15/)

[14] Ampoma, E.K.A., Rao, T.R. Labay, V.A., 2009 "Capacity \& performance issues in a MIMO based MB-OFDM ultrawide band communication system", Adaptive Science \& Technology, 2nd International Conference, IEEE, pp. 432, Accra

[15]Liu, H., Qiu, R. C., Tian, Z., 2005 "Error performance of pulse-based ultra wideband MIMO systems over indoor wireless channels," IEEE Wireless Communication conference. vol.4, pp. 2939-2944

[16] Proakis G. "Digital Communication, 2000" Fourth Edition, Irwin/McGraw-Hill, an imprint of the Mcgraw-Hill Companies, Inc, New York, USA, 